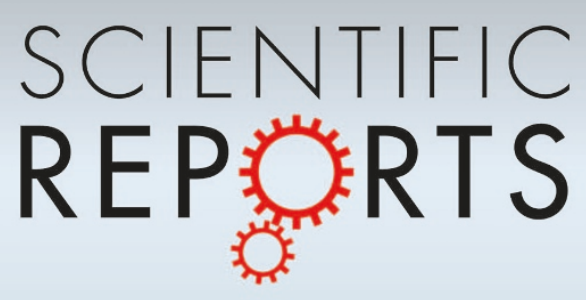

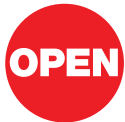

SUBJECT AREAS:

ELECTRICAL AND

ELECTRONIC

ENGINEERING

SENSORS AND BIOSENSORS

ELECTRONIC PROPERTIES AND

MATERIALS

APPLIED PHYSICS

Received

30 August 2012

Accepted

15 January 2013

Published

30 January 2013

Correspondence and requests for materials should be addressed to G.B. Ig.barillaro@iet. unipi.it)

\section{Addressing Reliability and Degradation of Chemitransistor Sensors by Electrical Tuning of the Sensitivity}

\author{
G. M. Lazzerini, L. M. Strambini \& G. Barillaro
}

Dipartimento di Ingegneria dell'Informazione: Elettronica, Informatica, Telecomunicazioni, Via G. Caruso 16, 56122 Pisa (Italy)

Here we show that electrical tuning of the sensitivity of chemitransistor sensors, namely

field-effect-transistors (FETs) exploiting nano/mesostructured sensing materials, can be used to effectively address two chief problems of state-of-the-art gas sensors, specifically fabrication reliability and degradation by aging. Both experimental evidences and theoretical calculations are provided to support such a result, using as a case-of-study junction field-effect-transistors (JFETs) exploiting mesostructured porous silicon (PS) as sensing material (PSJFETs) for the detection of nitrogen dioxide $\left(\mathrm{NO}_{2}\right)$ at hundreds ppb. Proof of concept is given by fully compensating the effect of fabrication errors on the sensitivity of two PSJFETs integrated on the same chip, which, though identical in principle, feature sensitivities to $\mathrm{NO}_{2}$ differing from about $30 \%$ before compensation. Although here-demonstrated for the specific case of PSJFETs, the concept of sensor reliability/aging problem compensation by sensitivity electrical-tuning can be applied to other chemitransistor sensors that exploit sensing materials different than PS.

N $\mathrm{O}_{2}$ is one of the most dangerous air-pollutants affecting human health and safety already at concentrations of a few part-per-million $(\mathrm{ppm})^{1}$. The need for miniaturized, sensitive, and reliable sensors for $\mathrm{NO}_{2}$ detection, in particular, and for air-quality monitoring, in general, has significantly pushed the research in the field in last years. Besides the well-known "chemiresistor" concept ${ }^{2-4}$, the "chemitransistor" concept, based on changes of the current of a FET structure that exploits a meso/nanostructured material as extra gate electrode, is an interesting alternative for gas sensors, in general, and $\mathrm{NO}_{2}$ detection, in particular ${ }^{5-7}$. A number of meso/ nanostructured materials, such as organic polymers ${ }^{8,9}$, porous silicon ${ }^{10,11}$, silicon nanowires ${ }^{12,13}$, carbon nanotubes ${ }^{14,15}$, and metal-oxide ${ }^{16}$ nanowires ${ }^{17}$, have been successfully used. The key advantage of chemitransistors over chemiresistors is the amplified sensor response due to current modulation by the extra gate electrode ${ }^{18}$, which allows sensitivity and limit of detection to be improved up to the part-per-billion (ppb) range for $\mathrm{NO}_{2}$ detection. Moreover, modulation of the extra gate voltage of chemitransistors has been demonstrated to allow tuning of the sensitivity to be effectively performed ${ }^{19-24}$. Nonetheless, aging effects and fabrication reliability, which lead to sensor sensitivity degradation over time and to different sensitivity values among identical (in principle, at least) sensors, respectively, still remain challenging for chemitransistor sensors, as well as for chemiresistor sensors, and represent a major bottleneck towards on-field applications.

In recent years there has been an intense research into self-healing and self-repairing materials (mostly polymer-based systems) capable of autonomously restoring either electrical or mechanical (or both of them in some cases) properties ${ }^{25-27}$. These smart materials are tremendously useful for enhancing structural safety, increasing lifetime, and improving environmental sustainability, with significant impact over a broad range of applications, from biology to electronics. As to electronics, self-healing digital circuits that rely on hardware redundancy or self-tuning digital circuits able to adapt circuit parameters in real-time have been proposed so far for addressing reliability problems and aging effects, respectively $y^{28,29}$.

Here we show, for the first time to our best knowledge, that real-time tuning of the sensitivity of chemitransistor sensors by controlling the electrical bias of the transistor gate-terminal can be used to effectively address two chief problems of state-of-the-art gas sensors, namely fabrication reliability and degradation by aging. Both experimental evidences and theoretical calculations are provided to support such a result, using as a case of study junction fieldeffect transistors (JFETs) with a mesostructured porous silicon (PS) extra gate (PSJFETs) for the room-temperature detection of nitrogen dioxide $\left(\mathrm{NO}_{2}\right)$ in the range of hundreds ppb. Proof of concept is given by fully compensating the effect of fabrication errors on the sensitivity of two PSJFETs integrated on the same chip, which, though identical in principle, result to have sensitivities to $\mathrm{NO}_{2}$ that differ by about $30 \%$ before compensation. 
This paper clearly demonstrates that concepts of self-repairing (self-tuning) materials and electronics can be successfully applied to sensor design, thus envisaging a novel class of self-tuning gas sensors with increased lifetime, enhanced reliability, and improved environmental sustainability.

\section{Results}

The PSJFET sensor is a $p$-channel JFET ${ }^{30}$ modified with a mesostructured porous silicon (PS) layer that is integrated on top of the $p$ channel between the source (S) and drain (D) terminals (Fig. 1a) ${ }^{31}$. PS is a nano/mesostructured form of silicon, obtained by electrochemical erosion of crystalline silicon in acidic solutions under anodic polarization, that features peculiar characteristics very appealing for gas sensing applications, among which huge surface-to-volume ratio (over $500 \mathrm{~m}^{2} / \mathrm{cm}^{3}$ ), effective room temperature interaction with gas molecules, and compatibility with commercial silicon-based integrated processes ${ }^{11}$. The PSJFET sensor is characterized by two independent gates: a solid electrical gate $(\mathrm{G})$, which allows the current of the JFET device to be electrically tuned, and a mesostructured sensing gate, which allows the JFET device to be provided with sensing capability. The two gates, electrical and sensing, independently

(a)
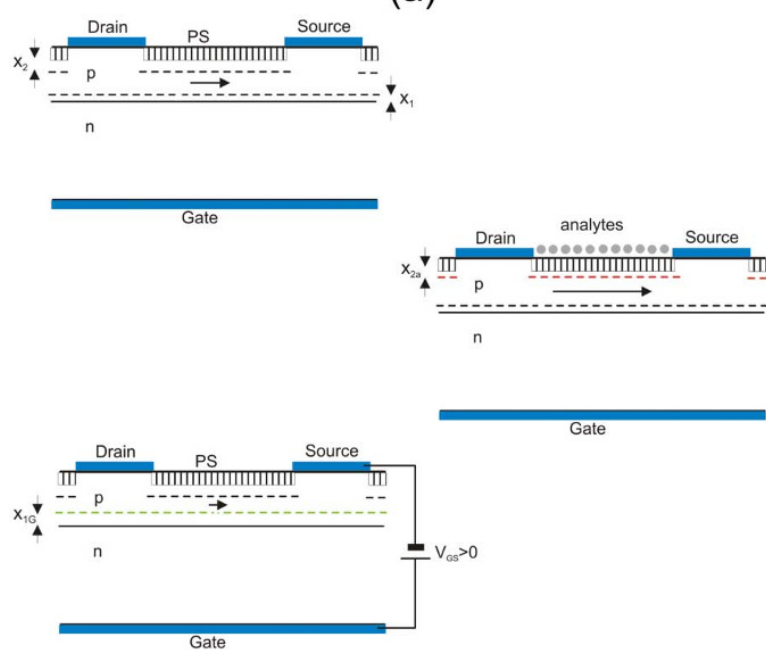

(c)

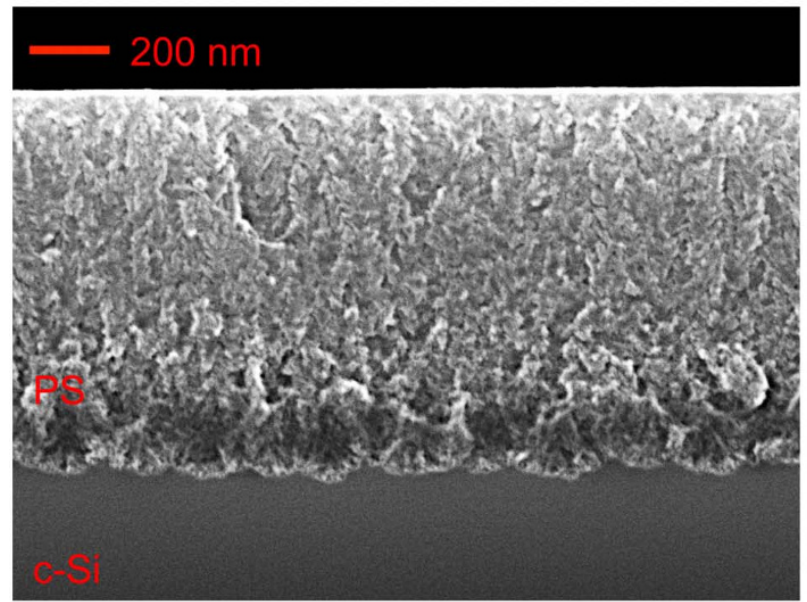

operate on the depletion regions (also known as space charge regions because they are free of mobile charge carriers and only contain fixed charges) $x_{1}$ at the $p$-channel $/ n$-silicon interface and $x_{2}$ at the PS $/ p$ channel interface, respectively, thus affecting the JFET current (Fig. 1a). On the one hand, the electrical gate allows the depletion region width $x_{1}$ at the $p$-channel $/ n$-silicon interface to be electrically tuned, thus effectively modulating the $p$-channel thickness and, in turn, the current flowing in the $p$-channel independently of the analyte concentration (Fig. 1a and 2a). On the other hand, the sensing gate allows the depletion region width $x_{2}$ at the PS/ $p$-channel interface to be tuned upon adsorption of analytes in the PS layer itself, thus effectively changing the $p$-channel thickness and, in turn, the current flowing in the $p$-channel dependently on the analyte concentration, for a given gate voltage (Fig. 1a and $2 b$ ).

The PSJFET sensor is fabricated by defining aluminum source and drain comb-finger contacts on a silicon substrate, this latter consisting of a 2.4- $\mu \mathrm{m}$-thick $p$-type silicon layer on top of a $n$-type silicon substrate, then performing the selective electrochemical erosion of part of the $p$-type silicon between drain and source contacts to produce the mesostructured PS layer on top of the $p$-channel ${ }^{31}$. The electrochemical erosion step is controlled to obtain mesostructured (b)

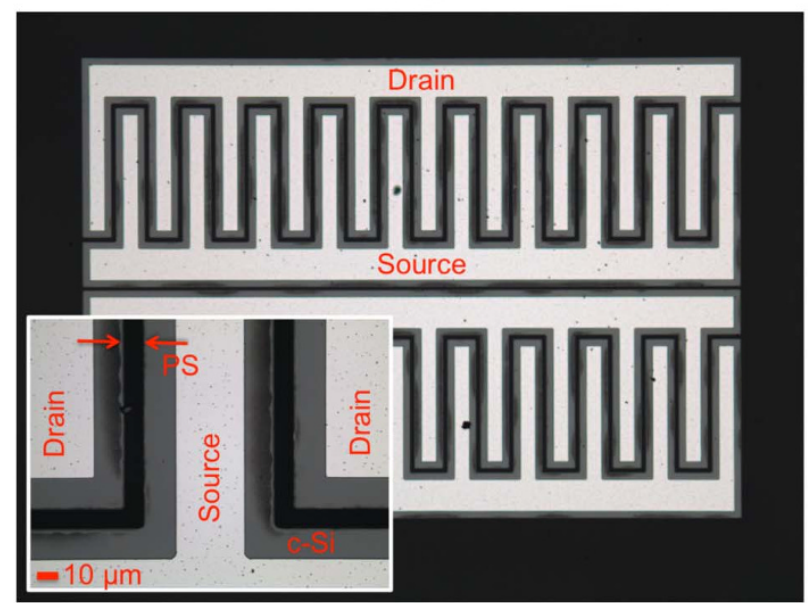

(d)

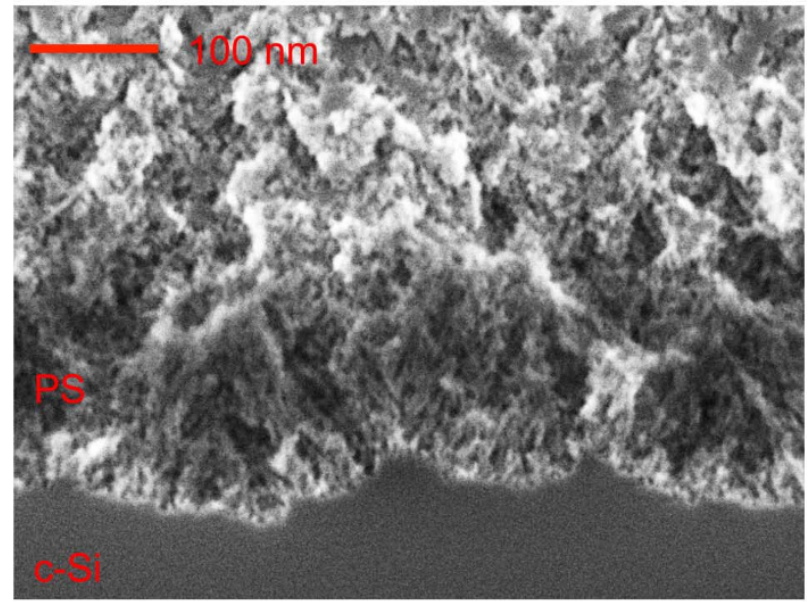

Figure $1 \mid$ (a) Schematic cross-section of the PSJFET sensor and effect of the gate voltage and analytes adsorbed into the PS on the depletion regions $x_{1}$ and $x_{2}$, respectively; (b) Optical microscope top-image of two PSJFET sensors highlighting source and drain interdigitated contacts, and (inset) magnification of one of the sensors in which the PS layer between contacts is clearly visible; (c) typical SEM cross-section image of the PS layer integrated between drain and source terminals of the PSJFET; (d) high-magnification SEM image of the PS layer in (c) highlighting the mesostructured morphology of the sensing gate. 
(a)

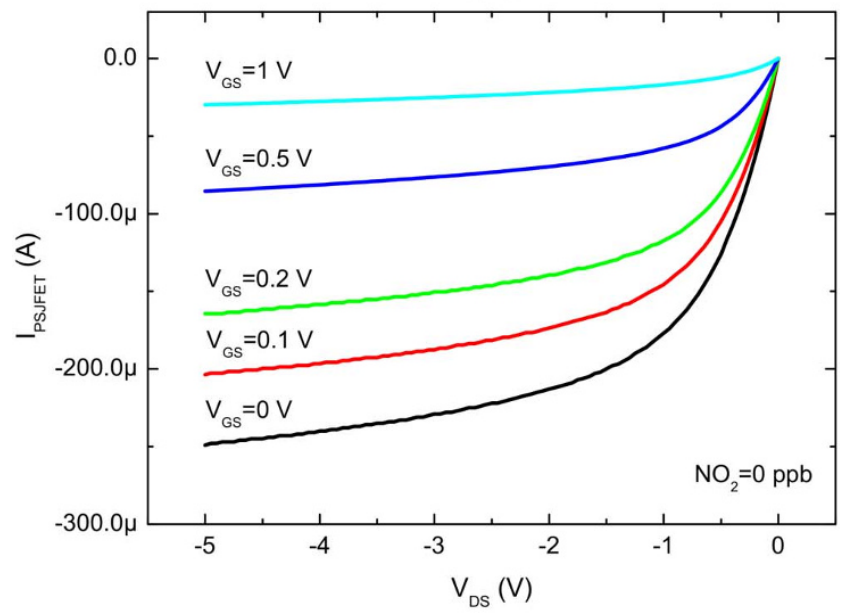

(b)

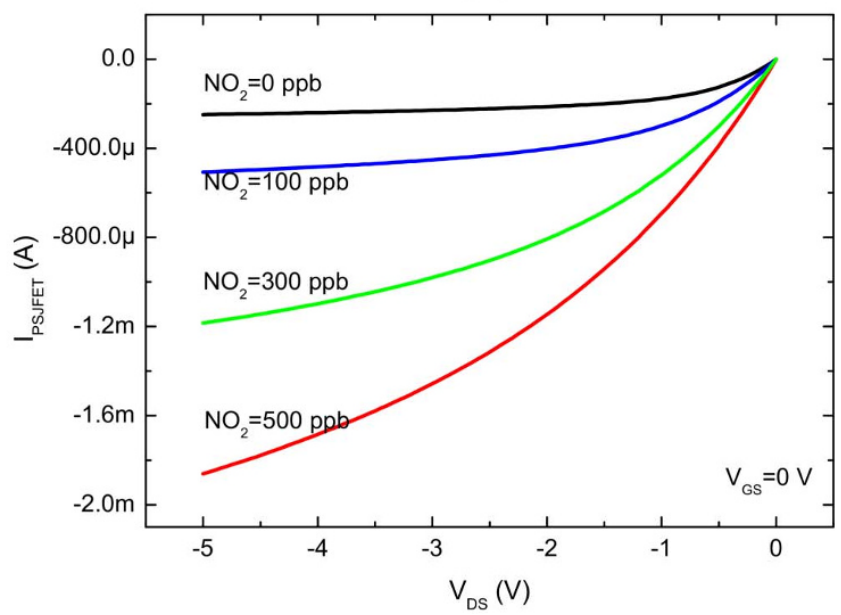

Figure $2 \mid$ (a) Typical $I_{P S J F E T}-V_{D S}$ output curves of the PSJFET in synthetic air for several gate voltages and (b) typical $I_{P S J F E T}-V_{D S}$ output curves of the PSJFET at $V_{G S}=0 \mathrm{~V}$ upon exposure to different $\mathrm{NO}_{2}$ concentrations.

PS layers with uniform thickness $t_{P S}$, which can be tuned between 800 $\mathrm{nm}$ and $1200 \mathrm{~nm}$. Consequently, the thickness of the crystalline $p$ channel underneath the PS layer ranges between $1600 \mathrm{~nm}$ and $1200 \mathrm{~nm}$. Fig. 1b shows an optical microscope image of two PSJFETs, together with details (in inset) of the PS layer between source and drain terminals. Fig. 1c and 1d show typical Scanning Electron Microscope (SEM) cross-section images of the mesostructured PS layer integrated between drain and source terminals of the PSJFET. In Fig. 1d, which was acquired at high magnifications $(500 \mathrm{kx})$, the mesostructured morphology of the PS layer (sensing gate) is clearly visible.

The PSJFET sensors are tested with $\mathrm{NO}_{2}$ at concentrations in the range $0-500 \mathrm{ppb}$, using the flow-through technique. For all the experiments, flow-rate is maintained at 200 standard cubic centimeter per minute ( $\mathrm{sccm}$ ), synthetic air is used as carrier gas, relative humidity is set to zero, and temperature is set to $30^{\circ} \mathrm{C}$. Both timeresolved (current versus time) and static (current versus voltage) measurements are performed for different $V_{G S}$ values and $\mathrm{NO}_{2}$ concentrations on a large set of silicon chips, each one integrating several PSJFETs with different channel width $W$ and length $L$, so as to carry out a quantitative investigation of the PSJFET behavior both from electrical and sensing points of view.

Fig. $2 \mathrm{a}$ and $2 \mathrm{~b}$ show typical output curves of the PSJFET, that is $I_{P S J F E T}$ versus $V_{D S}$ for different $V_{G S}$ values and $\mathrm{NO}_{2}$ concentrations, respectively. In the specific case of Fig. $2 \mathrm{a}$ and $2 \mathrm{~b}$, the output curves refer to a PSJFET sensor with channel length $L=10 \mu \mathrm{m}$, effective width $W=3.9 \mathrm{~mm}$, and PS thickness $t_{P S}=800 \mathrm{~nm}$. The output curves of the PSJFET sensor exhibit the typical behavior of a JFET device. For a given $V_{G S}$ value and $\mathrm{NO}_{2}$ concentration, the increase of the absolute value of the $V_{D S}$ voltage initially produces a linear increase of the absolute value of the sensor current $I_{\text {PSJFET }}$ (linear region); by increasing the absolute value of $V_{D S}$ beyond the linear region a saturation of the sensor current $I_{P S J F E T}$ is observed (saturation region), especially evident in synthetic air and for the lower $\mathrm{NO}_{2}$ concentrations. The effect of increasing $V_{G S}$ is that of reducing the sensor current $I_{P S J F E T}$ for any $V_{D S}$ value, while only slightly increasing the output resistance (slope of the curves in the saturation region slightly decreasing) of the PSJFET sensor (Fig. 2a). The effect of increasing the $\mathrm{NO}_{2}$ concentration is that of significantly increasing the sensor current $I_{P S J F E T}$ for any $V_{D S}$ value, while reducing the output resistance (slope of the curves in the saturation region increasing) of the PSJFET sensor, especially at the higher $\mathrm{NO}_{2}$ concentrations (Fig. 2b). In fact, the PS layer, which acts as a resistor in parallel to the $p$-channel of the JFET, has a conductivity whose value increases with the $\mathrm{NO}_{2}$ concentration ${ }^{32}$.
According to the literature, $\mathrm{NO}_{2}$ molecules act as strong acceptors at PS surface, thus producing an increase of the hole concentration (conductivity) of PS after adsorption of $\mathrm{NO}_{2}$ molecules. For a given $V_{G S}$, especially at the higher $\mathrm{NO}_{2}$ concentrations, the increase of the conductivity of the PS layer significantly reduces PS resistance value and, in turn, the output resistance of the PSJFET sensor, thus accounting for non-saturation of the sensor current $I_{P S J F E T}$.

Fig. 3a shows the typical relative current variation $\Delta I_{R}$ of a PSJFET as a function of the $\mathrm{NO}_{2}$ concentration for several $V_{G S}$ values, both in the linear $\left(V_{D S}=-0.1 \mathrm{~V}\right)$ and saturation $\left(V_{D S}=-3 \mathrm{~V}\right)$ regions (by

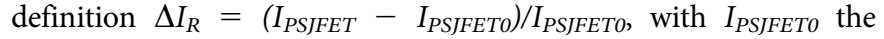
PSJFET sensor current in synthetic air). For a given $V_{G S}$ value, the value of $\Delta I_{R}$ linearly increases as a function of the $\mathrm{NO}_{2}$ concentration $\left(\left[\mathrm{NO}_{2}\right]\right)$ in the range $0-500 \mathrm{ppb}$, at least in a first-order approximation, in both the working regions. Moreover, for a given $\mathrm{NO}_{2}$ concentration, the higher the $V_{G S}$ value the larger the value of $\Delta I_{R}$. Fig. 3b shows the PSJFET sensitivity, which is defined as $S=\partial \Delta I_{R} /$ $\partial\left[\mathrm{NO}_{2}\right]$, for different $V_{G S}$ values, both in the linear and saturation regions. The value of $S$, for a given $V_{G S}$ value, is evaluated from the slope of the linear function obtained by best-fitting the corresponding experimental curve in Fig. 3a. Remarkably, Fig. 3b clearly highlights that the PSJFET sensitivity can be effectively varied over a large dynamic range (up to about one order of magnitude) by properly modulating the electrical gate voltage $V_{G S}$. The higher the $V_{G S}$ value the higher the sensitivity $S$, in both the working regions. Although the dynamic range of the PSJFET sensitivity does not significantly change between the two working regions, sensitivity values are larger in the saturation region than in the linear region, for any $V_{G S}$ value. For instance, by changing the $V_{G S}$ value between $0 \mathrm{~V}$ and $1 \mathrm{~V}$ the sensitivity $S$ of the PSJFET in Fig. 3a can be varied of a factor 7.6, from $0.1 \mathrm{ppb}^{-1}$ to $0.76 \mathrm{ppb}^{-1}$, in the saturation region, compared to a variation of a factor 7 , from $0.04 \mathrm{ppb}^{-1}$ to $0.28 \mathrm{ppb}^{-1}$ in the linear region. Fig. $3 \mathrm{~b}$ also shows best-fitting of experimental sensitivity data with a quadratic function, which allows to speculate the dependence of the PSJFET sensitivity on the square value of gate voltage $V_{G S}$ for both the working regions, at least in the range of $\mathrm{NO}_{2}$ concentrations under investigation. It is worth noting that the sensitivity tuning process does not produce any significant increase of the sensor power dissipation $P_{D}\left(P_{D}=V_{D S} I_{P S J F E T}+V_{G S} I_{G S}\right)$ when the gate voltage is increased. In fact, thanks to the high-impedance of the gate terminal of JFET devices and, in turn, to the very low current $I_{G S}$ (always much lower than $I_{P S I F E T}$ ) flowing through the gate terminal, the term $V_{G S} I_{G S}$ is always much smaller than $V_{D S} I_{P S J F E T}$, which mainly accounts for the sensor power dissipation. 
(a)

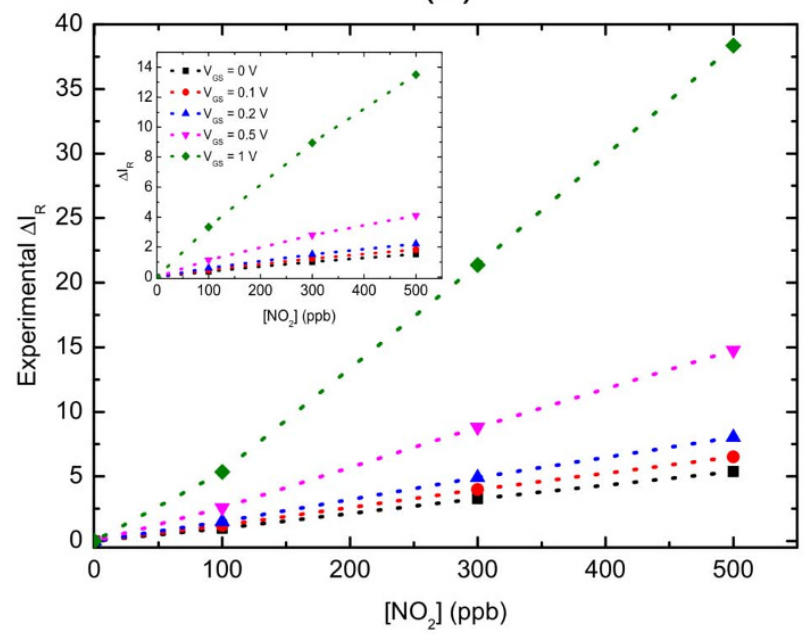

(c)

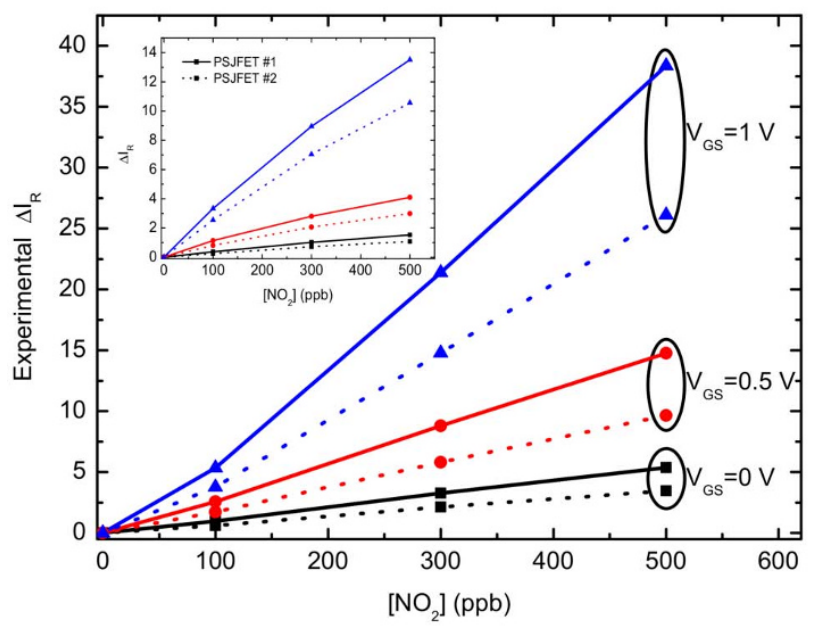

(b)

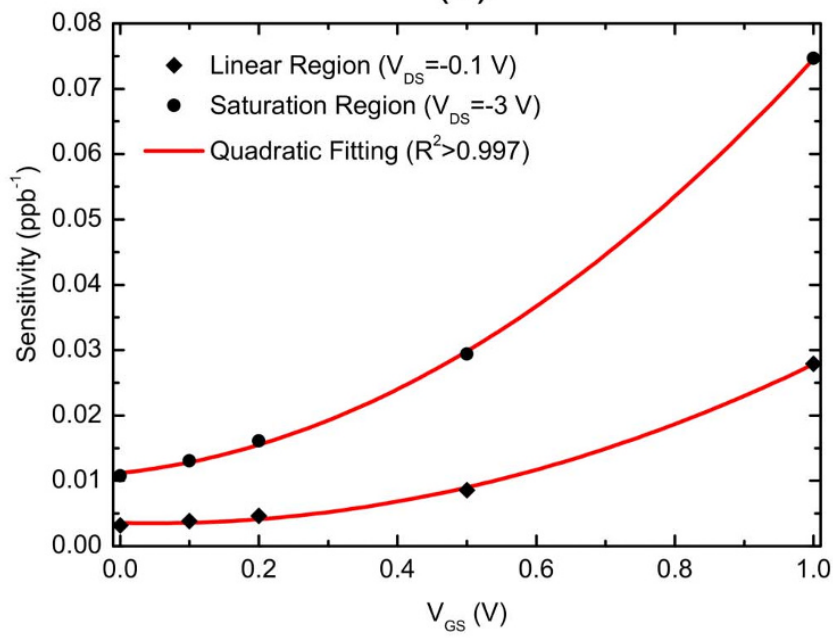

(d)

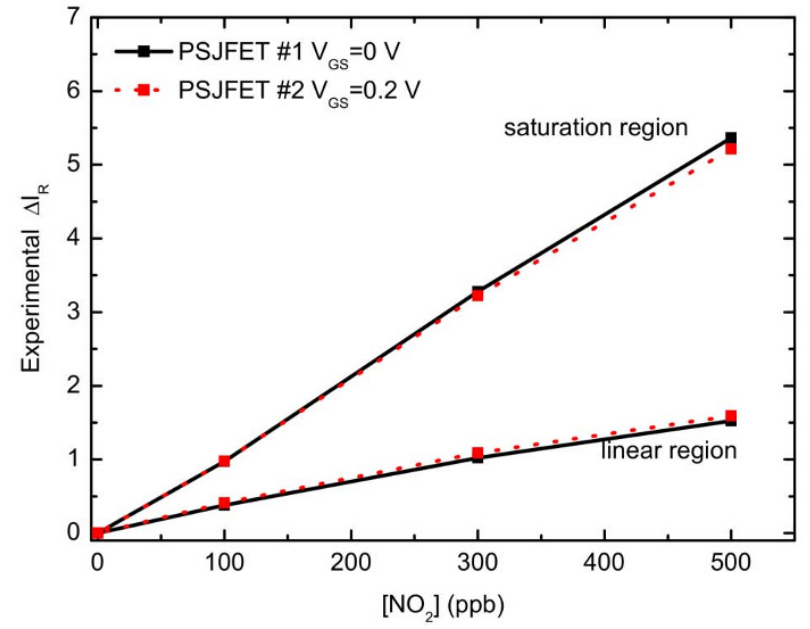

Figure $3 \mid$ (a) PSJFET experimental relative current variation, $\Delta I_{R}$, for several $\mathrm{NO}_{2}$ concentrations $(0-500 \mathrm{ppb})$, at different $V_{G S}$ voltages $(0-1 \mathrm{~V})$, measured in the saturation $\left(V_{D S}=-3 \mathrm{~V}\right)$ and (inset) linear $\left(V_{D S}=-0.1 \mathrm{~V}\right)$ regions; (b) Sensitivity $S$ of the PSJFET to $\mathrm{NO}_{2}$ versus gate voltage $V_{G S}$ in the linear and saturation regions; (c) Experimental $\Delta I_{R}$ for several $\mathrm{NO}_{2}$ concentrations $(0-500 \mathrm{ppb})$, at different $\mathrm{V}_{\mathrm{GS}}$ voltages $(0-1 \mathrm{~V})$, measured in the saturation and (inset) linear regions for two namely identical PSJFETs integrated on the same chip; (d) experimental electrical compensation of the sensitivity of PSJFET \#2, for both the linear and saturation regions, obtained by increasing the gate voltage $V_{G S}$ of PSJFET \#2 from $0 \mathrm{~V}$ to $0.2 \mathrm{~V}$ so as to achieve the superposition of $\Delta I_{R^{-}}\left[\mathrm{NO}_{2}\right]$ curves for the two PSJFETs of Fig. 3(c).

Fig. $3 \mathrm{c}$ shows the relative current variation $\Delta I_{R}$ of two PSJFETs as a function of the $\mathrm{NO}_{2}$ concentration for several $V_{G S}$ values, both in the linear $\left(V_{D S}=-0.1 \mathrm{~V}\right)$ and saturation $\left(V_{D S}=-3 \mathrm{~V}\right)$ regions. The two PSJFETs, namely PSJFET \#1 and \#2, are simultaneously integrated on the same silicon chip and are in principle identical. However, due to unavoidable fabrication process tolerances, the sensitivity of the two sensors toward $\mathrm{NO}_{2}$ results to be different, as it is clear from the calibration curves of Fig. $3 \mathrm{c}$. In particular, the sensitivity value of the PSJFET \#2 is always lower than that of the PSJFET \# 1 (see slope of the curves in Fig. 3c), for any $V_{G S}$ voltage. The reduction of the sensitivity of PSJFET \#2 with respect to PSJFET \#1 can be fully compensated in real-time by properly setting the gate operation voltage $V_{G S}$ of the PSJFET \#2, so as to match the sensitivity value of the PSJFET \#1. Fig. $3 \mathrm{~d}$ shows the experimental proof of electrical compensation of the sensitivity value of PSJFET \#2 to that of PSJFET \# 1 biased at $V_{G S}=0 \mathrm{~V}$. For instance, at $V_{G S}=0 \mathrm{~V}$, the two sensors feature sensitivity values that differ by about $30 \%$ before compensation (see curves for $V_{G S}=0 \mathrm{~V}$ in Fig. 3c). By increasing the $V_{G S}$ value of PSJFET \#2 from $0 \mathrm{~V}$ to $0.2 \mathrm{~V}$, the difference in sensitivity is fully compensated, both in the linear and saturation regions, as it is clear from the superposition of the $\Delta I_{R^{-}}\left[\mathrm{NO}_{2}\right]$ curves in Fig. $3 \mathrm{~d}$ over the whole range of $\mathrm{NO}_{2}$ concentrations.

To the best of our knowledge, this is the first report demonstrating that chief issues of up-to-date gas sensors, such as fabrication reliability and aging effects, can be effectively addressed by adjusting the sensitivity of sensors in real-time. As proof of concept, in this work compensation of the effect of fabrication tolerances on the sensitivity of two PSJFETs integrated on the same chip is demonstrated. In fact, though identical in principle, the two PSJFETs feature different sensitivities to $\mathrm{NO}_{2}$ before compensation, at a given $V_{G S}$ value, due to slightly different PS thicknesses. Electrical tuning of the sensitivity of one of the PSJFETs through $V_{G S}$ allows the effect of fabrication tolerances to be fully compensated, so that the two sensors feature same sensitivity values after compensation. Once compensation of fabrication tolerances of gas sensors by sensitivity tuning is successfully demonstrated, compensation of sensitivity degradation with aging by electrical tuning of the gate voltage is straightforward. In fact, sensitivity degradation by aging can be addressed likewise sensitivity variation due to fabrication tolerances, at least until the sensor is able to reach a saturation over time. After the PSJFET sensor is 
(a)

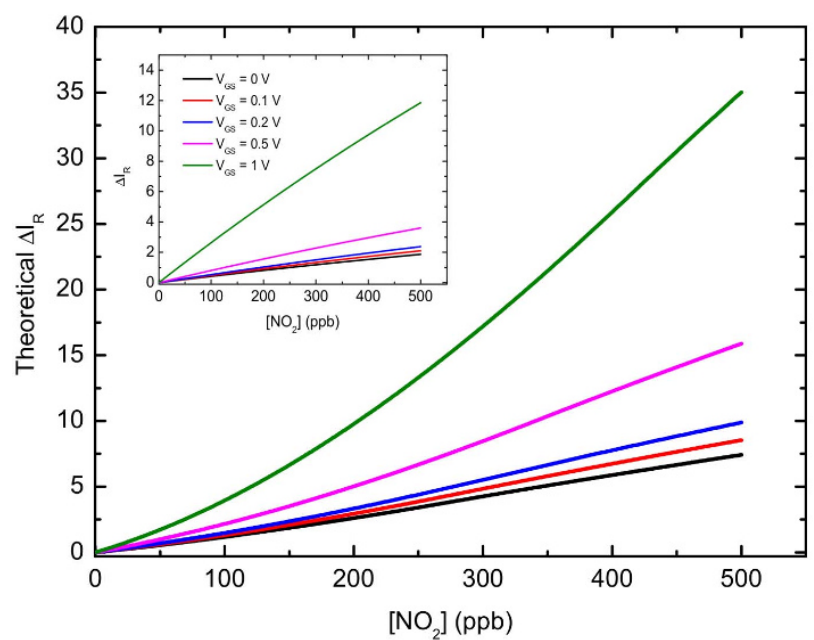

(b)

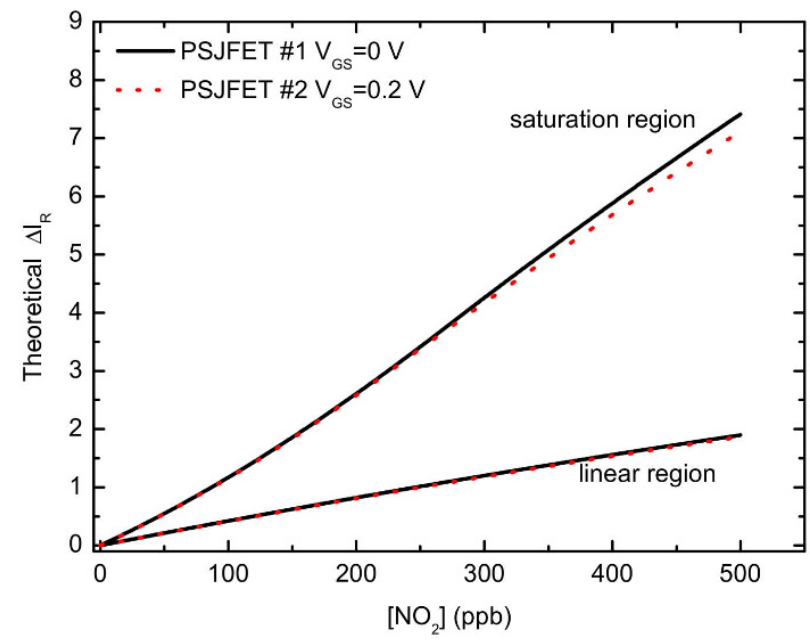

Figure $4 \mid$ (a) Theoretical $\Delta I_{R}$ as a function of the $\mathrm{NO}_{2}$ concentration $(0-500 \mathrm{ppb})$ for different $V_{G S}$ values $(0-1 \mathrm{~V})$, calculated for the PSJFET of Fig. 3a both in the saturation $\left(V_{D S}=-3 \mathrm{~V}\right)$ and (inset) linear $\left(V_{D S}=-0.1 \mathrm{~V}\right)$ regions; (b) Theoretical electrical compensation of the sensitivity of the PSJFET \#2 of Fig. $3 c$, for both the linear and saturation regions, obtained by increasing the gate voltage $V_{G S}$ of PSJFET \#2 from 0 V to $0.2 \mathrm{~V}$ so as to achieve the superposition of theoretical $\Delta I_{R}-\left[\mathrm{NO}_{2}\right]$ curves for the two PSJFETs of Fig. $3 \mathrm{c}$.

fabricated and initially calibrated, aging effects usually induce a reduction of the slope of the calibration curve (i.e. the sensitivity), being the saturation values for aged sensors generally different from those of the initial calibration curve, at the same gas concentrations. Sensitivity degradation over time due to aging effects can be then compensated likewise sensitivity variation due to fabrication tolerances by properly tuning the gate voltage of the PSJFET sensor.

A semi-analytical model ${ }^{10}$ of the PSJFET sensor is used to theoretically corroborate experimental results on the electrical tuning of the sensitivity by gate-voltage control. The model takes into account changes in both conductance $G_{P S}$ and fixed-charge density $Q_{P S}$ of the PS layer upon exposure to $\mathrm{NO}_{2}$. Under the assumption that these two effects give rise to two independent contributions to the sensor current, the PS is schematized with a resistance $R_{P S}=1 / G_{P S}=L /$ $\left(\sigma_{P S} \cdot W \cdot t_{P S}\right)$ (being $\sigma_{\text {PS }}$ the PS conductivity) operating in parallel to a JFET with a $p$-channel cross-section that is reduced by a factor $x_{2}=$ $Q_{P S} \cdot t_{P S} / q N_{A}$, being $q$ the elementary charge and $N_{A}$ the $p$-doping concentration. The current flowing into the PSJFET sensor $I_{P S J F E T}$ is therefore given by the sum of the current flowing into $R_{P S}\left(I_{P S}\right)$ and into the JFET $\left(I_{\text {JFET }}\right)$, both depending on the $\mathrm{NO}_{2}$ concentration. In addition, two resistors $R_{S}$ and $R_{D}$ in series to the JFET/ $R_{P S}$ branch are also included to model the silicon resistive paths from the external source (S) and drain (D) contacts to the PSJFET active region (right underneath the PS layer), and the contact resistances at the S and D terminals. An analytical equation can be written for $I_{P S J F E T}$ that theoretically describes the PSJFET electrical behavior as a function of both $V_{D S}$ and $V_{G S}$ voltages and $\mathrm{NO}_{2}$ concentration. This equation is used to perform the best-fitting of experimental current-voltage output curves measured for several $V_{G S}$ values at different $\mathrm{NO}_{2}$ concentrations so as to obtain quantitative data on $G_{P S}$ and $Q_{P S}$ as a function of the $\mathrm{NO}_{2}$ concentration in the range under investigation and, in turn, on the variation of $I_{P S}$ and $I_{J F E T}$ with $\mathrm{NO}_{2}$. Fig. S1 shows an example of theoretical (dotted lines) current-voltage output curves best-fitting the experimental (solid lines) curves, measured for one of the PSJFET used in this work. A good agreement between theoretical and experimental curves is clearly obtained for all tested $V_{G S}$ values and $\mathrm{NO}_{2}$ concentrations, thus validating the proposed semi-analytical model. Details of analytical equations describing the PSJFET and of the best-fitting procedure are provided in the section Methods, subsection Theoretical modeling and fitting.
Once $G_{P S}$ and $Q_{P S}$ as a function of the $\mathrm{NO}_{2}$ concentration are known from the best-fitting of experimental data (Fig. S2), the effect of PS thickness variations $\Delta t_{P S}$, due to fabrication tolerances, on the $I_{P S J F E T}$ current of different PSJFETs, integrated either on the same or different chips, can be easily calculated, being both $R_{P S}$ and $x_{2}$ depending on the thickness of the PS layer $t_{P S}$. Calculation of theoretical current-voltage output curves of PSJFETs with different PS thickness values is thus performed as a function of the $V_{G S}$ value, for $\mathrm{NO}_{2}$ concentrations in the range 0-500 ppb. Fig. 4a shows theoretical relative current variation $\Delta I_{R}$ of a PSJFET with PS thickness of $800 \mathrm{~nm}$ as a function of the $\mathrm{NO}_{2}$ concentration for different $V_{G S}$ values, in the linear $\left(V_{D S}=-0.1 \mathrm{~V}\right)$ and saturation $\left(V_{D S}=\right.$ $-3 \mathrm{~V})$ regions. The theoretical output curves well agree with the experimental curves measured for the PSJFET of Fig. 3a. By considering a PS thickness variation of $-1.5 \%$ for the PSJFET \# 1 and PSJFET \# 2 of Fig. 3c, according to SEM observation of the PSJFET cross-section, a theoretical analysis aimed at demonstrating sensitivity compensation of the two sensors through $V_{G S}$ tuning is carried out. Fig. $4 \mathrm{~b}$ shows theoretical compensation of the sensitivity value of PSJFET \# 2 to that of PSJFET \# 1 biased at $V_{G S}=0 \mathrm{~V}$. The compensation is obtained by increasing the gate-voltage value of the PSJFET \#2 at $0.2 \mathrm{~V}$, which allows a pretty perfect matching of the $\Delta I_{R^{-}}\left[\mathrm{NO}_{2}\right]$ curves of the two PSJFETs to be achieved, both in the linear and saturation regions. Fig. 4 b well agrees with experimental compensation data in Fig. 3d, thus strongly corroborating electrical sensitivity compensation/tuning of PSJFETs from a theoretical point of view.

In nanostructured chemitransistor gas sensors, the adjustment of the gate bias has been shown to induce a significant modulation of the chemical interactions that occur between sensing material and target analyte $\mathrm{e}^{20-24}$. Consequently, gate bias also affects the charge transfer processes occurring between adsorbates and the nanostructured material, thus causing a variation of the sensor response times besides sensitivity tuning ${ }^{20-24}$. Conversely to state-of-the-art nanostructured chemitransistor sensors, which are mostly based on MOS (Metal Oxide Semiconductor) FET $^{30}$ structures that feature a strong interaction between electrical and sensing (the nanostructured material) gates mostly because of the presence of the insulating layer (capacitor effect), in the case of the PSJFET sensor the two gates, electrical and sensing, independently operate on the depletion regions $x_{1}$ and $x_{2}$ at the $p$-channel $/ n$-silicon interface and the 
PS/p-channel interface, respectively. This has been demonstrated both with experimental data and theoretical analyses (see Ref. 10 and section Methods, subsection Theoretical modeling and fitting, of this manuscript). In the PSJFET sensor the voltage $V_{G S}$ applied between gate and source terminals mostly drops across the depletion region $x_{1}$, at the $p$-channel $/ n$-silicon interface, with two main advantages. The former is an efficient modulation of the width of the depletion region $x_{1}$ with the $V_{G S}$ value (typical of Junction FET structures), which allows the PSJFET sensitivity to be tuned with high efficacy. The latter is an effective minimization of the electrical interaction between gate terminal and sensing material, which allows to strongly reduce the effect of the gate voltage on the chemical interaction occurring between adsorbates and nanostructured material.

In order to experimentally investigate the dependence of the PSJFET response times on the $V_{G S}$ voltage values, several PSJFET sensors able to work with a large dynamic range of $V_{G S}$ values, from 0 to $10 \mathrm{~V}$, are fabricated with a smaller PS thickness with respect to PSJFET \#1 and \#2. They are continuously tested for several days by monitoring the sensor current over time, in the linear region $\left(V_{D S}=\right.$ $-0.1 \mathrm{~V}$ ) at constant $\mathrm{NO}_{2}$ concentration $(500 \mathrm{ppb})$, for different $V_{G S}$ values $(0 \mathrm{~V}, 1 \mathrm{~V}$, and $10 \mathrm{~V})$. Typical time-resolved curves of the PSJFET current over time for the different tested $V_{G S}$ values are reported in Fig. 5a and 5b for two different PSJFETs (\#3 and \#4) integrated on the same chip. All the tested sensors show a reliable behavior independently of the gate voltage values, with the sensor current (absolute value) quickly increasing when $\mathrm{NO}_{2}$ is injected in the test chamber to reach the saturation value in a few minutes, and quickly decreasing when $\mathrm{NO}_{2}$ is removed from the test chamber to restore the quiescent current value. The quiescent current value (in synthetic air) of PSJFET \#3 and \#4 is significantly larger than that of PSJFET \# 1 and \#2, and the absolute (and relative) current variation is smaller, due to the smaller PS thickness and, in turn, higher doping concentration of the $p$-channel (see Eq. 3 of section Methods, subsection Theoretical modeling and fitting) of PSJFET \#3 and \#4, compared to PSJFET \#1 and \#2, needed to ensure a larger dynamic $V_{G S}$ range (up to $10 \mathrm{~V}$ ) of investigation. A slight drift of the quiescent current over time is observed for all the tested sensors. Fig. $5 \mathrm{c}$ shows both rise and fall times (mean value and standard deviation) of PSJFET \#3 and \#4 for each tested $V_{G S}$ value. A maximum variation of about $20 \%$ and $15 \%$ for the fall and rise time, respectively, is measured over a $V_{G S}$ variation from $0 \mathrm{~V}$ to $10 \mathrm{~V}$. Note that such a high variation of the gate voltage, up to $10 \mathrm{~V}$, is usually neither necessary nor exploited for the sensitivity tuning process, for which a variation of the gate voltage from $0.1 \mathrm{~V}$ to $1 \mathrm{~V}$ (at least one order of magnitude below $10 \mathrm{~V}$ ) is usually adequate. If the $V_{G S}$ variability range is restricted to the voltage values from $0 \mathrm{~V}$ to $1 \mathrm{~V}$, then the effect of the gate voltage on the response times of the PSJFET is negligible, at least within the experimental error of performed measurements.

\section{Discussion}

Summarizing, in this work, electrical tuning of the sensitivity of porous silicon (PS) junction field-effect transistor (JFET) gas sensors by bias-control of the JFET gate-terminal is shown to effectively address two chief problems of solid-state gas sensors, namely fabrication reliability and aging effects, which represent a major bottleneck towards in-field applications. Proof of concept is given by fully compensating the effect of fabrication errors on the sensitivity of two PSJFETs integrated on the same chip, which, though identical in principle, feature different sensitivities to $\mathrm{NO}_{2}$ (about 30\%) before compensation. The sensitivity tuning process does not significantly affect the response times of the PSJFETs over a large range $(0 \mathrm{~V}-$ $10 \mathrm{~V}$ ) of gate voltage. Although fabrication tolerance compensation of chemitransistor sensors by sensitivity tuning is here demonstrated for the specific case of the PSJFET, the general concept can be in

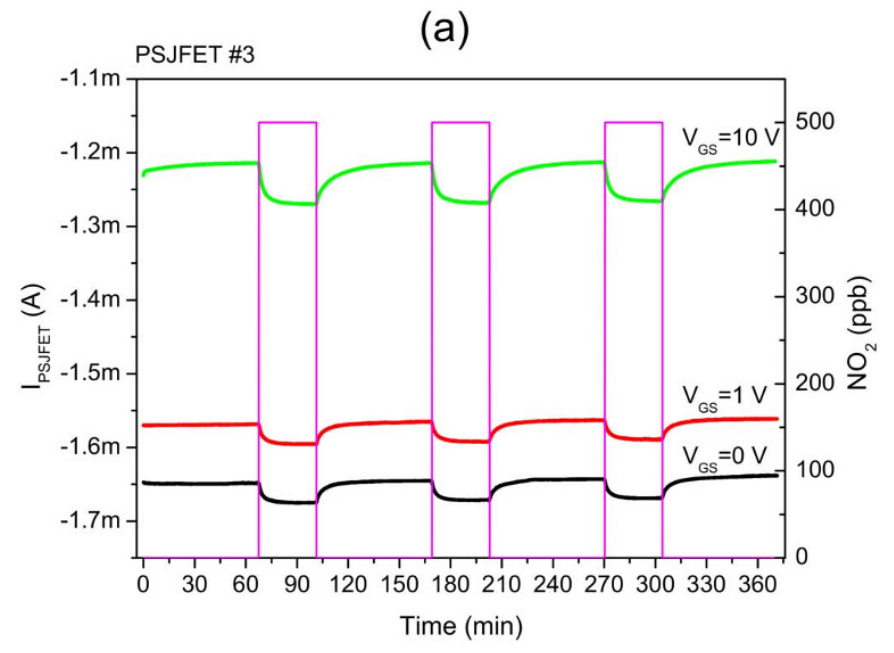

(b)

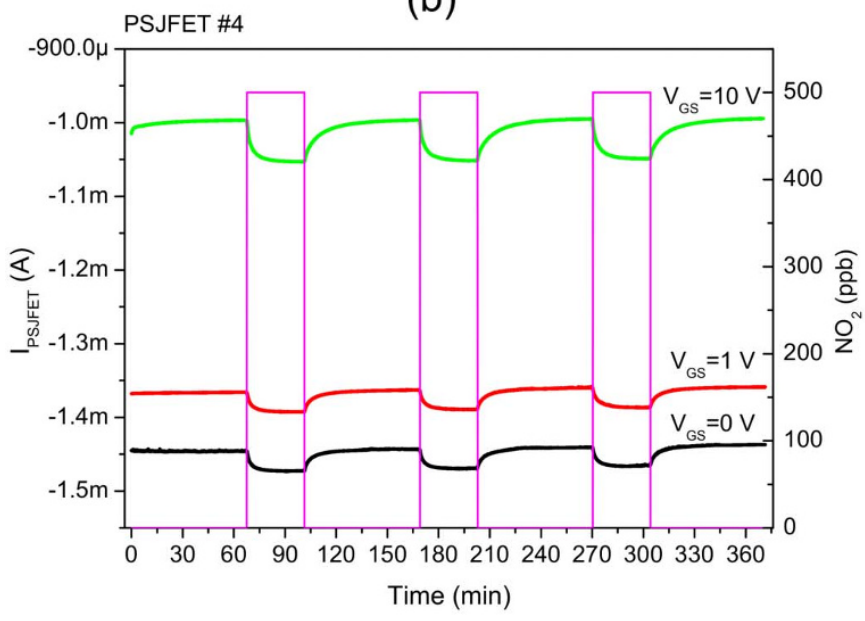

(c)

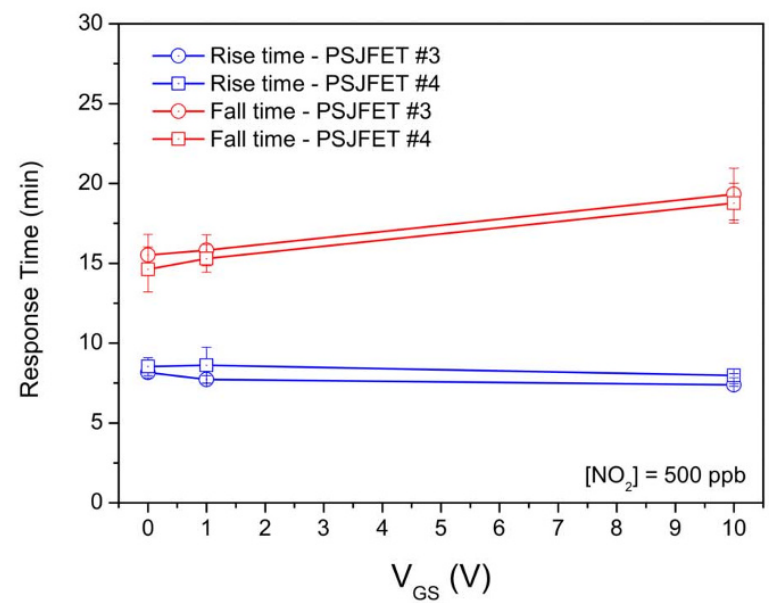

Figure $5 \mid$ (a, b) Time-resolved curves (current versus time) for two PSJFETs biased in the linear region $\left(V_{D S}=-0.1 \mathrm{~V}\right)$ upon exposure to $\mathrm{NO}_{2}$ at concentration of $500 \mathrm{ppb}$, for different gate voltage values $\left(V_{G S}=0 \mathrm{~V}\right.$, $1 \mathrm{~V}, 10 \mathrm{~V})$; (c) rise and fall times of the two PSJFETs in (a) and (b) as a function of the gate voltage values in the range from $V_{G S}=0 \mathrm{~V}$ to $V_{G S}=10 \mathrm{~V}$.

principle applied to other chemitransistor sensors that exploit sensing materials different from porous silicon.

This work breaks a new ground in the gas sensor arena by demonstrating that concepts of self-repairing (self-tuning) materials and 
electronics can be successfully applied to sensor design, thus envisaging a novel class of self-tuning gas sensors with increased lifetime, enhanced reliability, and improved environmental sustainability.

\section{Methods}

Fabrication and experimental measurements. The fabrication process of the PSJFET consists of the following main steps: i) $n$-type silicon substrate with doping of $10^{15} \mathrm{~cm}^{-3}$; ii) boron implantation and diffusion to form a $p$-type layer, $2.4 \mu \mathrm{m}$ deep with a maximum surface doping of $10^{17} \mathrm{~cm}^{-3}$; iii) definition of aluminium interdigitated electrical contacts for drain and source terminals ( $1^{\text {st }}$ mask); iv) aluminium evaporation for electrical gate contact formation on the back of the chip; v) source and drain contacts protection by using a photoresist mask ( $2^{\text {nd }}$ mask) hardbaked at $140^{\circ} \mathrm{C}$ for 30 minutes, which is a crucial step for making the resist layer able to withstand the subsequent electrochemical etching (anodization); vi) selective anodization in aqueous HF-based solution of the $p$-type material, through the photoresist-free spaces, to form a PS layer with uniform thickness. Several PSJFET sensors with channel length $L=10 \mu \mathrm{m}$, different channel width, $W,\left(W_{1}=9.6 \mathrm{~mm}\right.$, $W_{2}=5.8 \mathrm{~mm}$, and $W_{3}=3.9 \mathrm{~mm}$ ) and same nominal PS thickness are integrated on the same chip. Several chips are prepared using the same anodization current density and only changing the anodization time in order to produce PSJFETs featuring PS layers with different chip-to-chip thicknesses, ranging from $800 \mathrm{~nm}$ and $1000 \mathrm{~nm}$, but same morphological mesostructured features. Each chip is then mounted on a TO12 metal-package and placed into a sealed chamber with temperature fixed at $30 \pm$ $0.5^{\circ} \mathrm{C}$ for testing.

The flow-through technique is used to expose the PSJFETs to a constant flux of 200 sccm alternating synthetic air and mixtures of synthetic air $\mathrm{NO}_{2}$ at different $\mathrm{NO}_{2}$ concentrations between $100 \mathrm{ppb}$ and $500 \mathrm{ppb}$. The sensors are exposed to $\mathrm{NO}_{2}$ mixtures for $30 \mathrm{~min}$ and then to synthetic air for $60 \mathrm{~min}$. Response time, which is defined as the time required for the sensor output to change from its previous state to a final settled value within a tolerance band of $\pm 10 \%$, ranges from a few minutes to several minutes at room temperature for $\mathrm{NO}_{2}$ adsorption and desorption cycles, respectively. Response times are estimated by monitoring the sensor current $I_{\text {PSJFET }}$ over time (up to a few days) for different $\mathrm{NO}_{2}$ concentrations, at constant $V_{D S}$ and $V_{G S}$ values. The effect of the electrical gate on the response time is evaluated by monitoring the sensor current $I_{\text {PSIFET }}$ over time (up to a few days) for constant $\mathrm{NO}_{2}$ concentration and $V_{D S}$ value, and different $V_{G S}$ values in the range from $0 \mathrm{~V}$ to $10 \mathrm{~V}$. PSJFETs with different $L$ and $W$ values show similar qualitative behavior and a quantitative behavior that is coherent with the ratio $W / L$, as expected.

Output current-voltage curves $I_{P S J F E T}-V_{D S}$ of the PSJFETs are measured using a source-measure unit (Keithley 2400), by sweeping the $V_{D S}$ value between $0 \mathrm{~V}$ and $-5 \mathrm{~V}$, with step of $-0.05 \mathrm{~V}$, and simultaneously monitoring the current flowing between the drain and source terminals, for a given $V_{G S}$ value. The effect of the electrical gate on the sensor current is measured by changing the $V_{G S}$ voltage between $0 \mathrm{~V}$ and $1 \mathrm{~V}$, with a minimum step of $0.1 \mathrm{~V}$. Once the response times of the sensors at room temperature are known from the time-resolved analysis, the measurement of the output current-voltage curves are carried out by exposing the sensors to $\mathrm{NO}_{2}$ mixtures for $30 \mathrm{~min}$ and then to synthetic air for $30 \mathrm{~min}$.

Theoretical modeling and fitting. The PSJFET electrical behavior is described by a set of equations that include the dependence on the $\mathrm{NO}_{2}$ concentration of the current flowing through the JFET and structure and the PS layer ${ }^{10}$.

As for the JFET structure, the current $I_{J F E T}$ can be modeled in the linear region by:

$$
I_{J F E T_{-} \text {lin }}=k_{1}\left(V_{D i}-V_{S i}\right)+(3 / 4) \mathrm{k}_{2}\left[\left(V_{b i}+V_{G}-V_{D i}\right)^{4 / 3}-\left(V_{b i}+V_{G}-V_{S i}\right)^{4 / 3}\right]
$$

where $V_{D i}$ and $V_{S i}$ are the drain and source voltages corrected of the voltage drop across the resistive paths $R_{D}$ and $R_{S}$ from external source and drain contacts to the JFET active region right underneath the PS layer, respectively, $V_{b i}$ is the built-in voltage at the $p n$ junction, $k_{2}$ is a constant that depends on the doping profile of the $p n$ junction, and $k_{1}$ is a parameter that takes into account the geometrical dimension of the $p$-channel and the effect of the adsorbed $\mathrm{NO}_{2}$ molecules on the depletion region $x_{2}$ at the PS/p-channel interface:

$$
k_{1}=\frac{W}{\rho L} \cdot\left(h-x_{2}\right)
$$

where $\rho$ is the resistivity of the channel, $W, L$, and $h$ are the width, length, and metallurgical height of the $p$-channel, respectively, and $x_{2}$ is given by:

$$
x_{2}=\frac{Q_{P S}\left(\left[N O_{2}\right]\right)}{q N_{A}} \cdot t_{P S}
$$

being $q$ the elementary charge, $N_{A}$ the $p$-doping concentration of the channel, and $t_{P S}$ the thickness of the PS layer. $Q_{P S}$ is the fixed-charge density in the PS layer and represents the charge distributed on the PS layer surface and here macroscopically schematized as a volume charge uniformly distributed in the PS layer.

The pinch-off occurs when, at the JFET drain (Di), the sum of the depletion regions, $x_{1}$ and $x_{2}$ equals $h$. This occurs when $V_{D i}$ reaches the value:

$$
V_{\text {Di_sat }}=V_{b i}+V_{G}-\left(k_{1} / k_{2}\right)^{3}
$$

Under this condition, the JFET current reaches its saturation value, which is independent of any further increase of the drain voltage:
$I_{J F E T_{-} s a t}=k_{1}\left(V_{D i_{-} s a t}-V_{S i}\right)+(3 / 4) k_{2}\left[\left(V_{b i}+V_{G}-V_{D i_{s} s a t}\right)^{4 / 3}-\left(V_{b i}+V_{G}-V_{S i}\right)^{4 / 3}\right](5)$

As for the current flowing through the PS layer $I_{P S}$, this is given by:

$$
I_{P S}=G_{P S}\left(\left[\mathrm{NO}_{2}\right]\right) \cdot\left(V_{D i}-V_{S i}\right)
$$

where $G_{P S}\left(\left[\mathrm{NO}_{2}\right]\right)=1 / R_{P S}\left(\left[\mathrm{NO}_{2}\right]\right)$, being $R_{P S}\left(\left[\mathrm{NO}_{2}\right]\right)$ the PS resistance.

Equations 1-6 are used to best-fit experimental output current-voltage curves for a number of PSJFETs, integrated both on the same and different chips, and extract quantitative information on the effect of $\mathrm{NO}_{2}$ on the sensor current through $G_{P S}, k_{1}$, and $k_{2}$ that are used as fitting parameters. For all the devices $R_{S}=R_{D}=25 \Omega$, which is estimated from the sensor geometrical dimensions and doping profiles. Fig $\mathrm{S} 1$ shows experimental output curves (solid lines) of a PSJFET for several $V_{G S}$ values at different $\mathrm{NO}_{2}$ concentrations superimposed to theoretical output curves (dotted lines) obtained by the best-fitting of experimental data. Fig. S2 $(\mathrm{a}-\mathrm{c})$ show mean values of $G_{P S}, k_{1}$, and $k_{2}$ as a function of the $\mathrm{NO}_{2}$ concentration, for the different $V_{G S}$ values, resulting from the best-fitting procedure of data in Fig. S1, with error bars representing one standard deviation. As expected, $k_{1}$ and $G_{P S}$ are independent of the gate voltage $V_{G S}$, though they significantly depend on the $\mathrm{NO}_{2}$ concentration. In particular, $k_{1}$ increases from $0.99 \times 10^{-3} \mathrm{~A} \cdot \mathrm{V}^{-1}$ for $\left[\mathrm{NO}_{2}\right]=0 \mathrm{ppb}$ to $1.38 \times$ $10^{-3} \mathrm{~A} \cdot \mathrm{V}^{-1}$ for $\left[\mathrm{NO}_{2}\right]=500 \mathrm{ppb} ; G_{P S}$ increases from $9.7 \times 10^{-6} \mathrm{~S}$ for $\left[\mathrm{NO}_{2}\right]=$ $0 \mathrm{ppb}$ to $1.67 \times 10^{-4} \mathrm{~S}$ for $\left[\mathrm{NO}_{2}\right]=500 \mathrm{ppb}$. On the contrary, $k_{2}$ only depends on the geometrical characteristics and doping profiles of the JFET structure, and its value is expected to be independent of both $\mathrm{NO}_{2}$ concentration and gate voltage $V_{G S}$. In fact, $k_{2}$ has a mean value of $7.9 \times 10^{-4} \mathrm{~A} \cdot \mathrm{V}^{3 / 4}$ for $\left[\mathrm{NO}_{2}\right]$ between $0 \mathrm{ppb}$ and $500 \mathrm{ppb}$, with a standard deviation of $0.2 \times 10^{-4} \mathrm{~A} \cdot \mathrm{V}^{3 / 4}$.

Equations 2 and 3 are then used to extract information on $x_{2}$ and, in turn, on $Q_{P S}$ as a function of the $\mathrm{NO}_{2}$ concentration. Remarkably, together with an increase of $G_{P S}$, the model confirms that $x_{2}$ reduces from $1.1 \mu \mathrm{m}$ for $\left[\mathrm{NO}_{2}\right]=0 \mathrm{ppb}$ to $0.9 \mu \mathrm{m}$ for $\left[\mathrm{NO}_{2}\right]=500 \mathrm{ppb}$, due to a reduction of the charge density $Q_{P S}$ in the PS layer from 1 $\times 10^{15} \mathrm{~cm}^{-3}$ for $\left[\mathrm{NO}_{2}\right]=0 \mathrm{ppb}$ to $8.1 \times 10^{14} \mathrm{~cm}^{-3}$ for $\left[\mathrm{NO}_{2}\right]=500 \mathrm{ppb}(\mathrm{Fig}$. S2 $(\mathrm{d}-$ e)). By best-fitting experimental data obtained for $G_{P S}$ and $Q_{P S}$ versus $\mathrm{NO}_{2}$ concentration with a second-order polynomial function the following equations are obtained:

$$
\begin{gathered}
G_{\mathrm{PS}}\left(\left[\mathrm{NO}_{2}\right]\right)=1.85 \cdot 10^{-10} \times\left[\mathrm{NO}_{2}\right]^{2}-2.26 \cdot 10^{-7} \times\left[\mathrm{NO}_{2}\right]+9.75 \cdot 10^{-6} \\
Q_{\mathrm{PS}}\left(\left[\mathrm{NO}_{2}\right]\right)=1.97 \cdot 10^{8} \times\left[\mathrm{NO}_{2}\right]^{2}-4.56 \cdot 10^{11} \times\left[\mathrm{NO}_{2}\right]+9.94 \cdot 10^{14}
\end{gathered}
$$

with $\left[\mathrm{NO}_{2}\right]$ in ppb (red curve in Fig. S2(a) and (d)).

Equations 7 and 8 are exploited to perform the quantitative evaluation of the effect of fabrication process tolerances, such as variation of the PS thickness with respect to the nominal value, on the sensitivity of PSJFET sensors. To this end, $t_{P S}$ is defined as $t_{P S}=t_{P S O}\left(1+\Delta t_{r e l}\right), \Delta t_{r e l}$ being the relative variation of the PS thickness with respect to the nominal value $t_{P S O}$. Being $Q_{P S}$ by definition independent of the thickness of the PS layer, $k_{1}$ and $G_{P S O}$ can be expressed as:

$$
\begin{gathered}
k_{1}=\frac{W}{\rho L} \times\left(h-\frac{Q_{P S}}{q N_{A}} \cdot t_{P S}\left(1+\Delta t_{r e l}\right)\right) \\
G_{P S}=G_{P S 0}\left(1+\Delta t_{r e l}\right)
\end{gathered}
$$

where $G_{P S O}$ is the PS conductance value at $\Delta t_{r e l}=0$.

1. Guerreiro, C., Larssen, S., de Leeuw, F. \& Foltescu, V. Air quality in Europe 2011 report. EEA Technical Report 12, (2011) (http://www.eea.europa.eu/ publications/air-quality-in-europe-2011) accessed 30th August 2012.

2. Jimenez-Cadena, G., Riu, J. \& Rius, F. X. Gas sensors based on nanostructured materials. The Analyst 132, 1083-1099 (2007)

3. Janata, J. \& Josowicz, M. Conducting polymers in electronic chemical sensors. Nature Materials 2, 19-24 (2003).

4. Tricoli, A., Righettoni, M. \& Teleki, A. Semiconductor Gas Sensors: Dry Synthesis and Application. Angewandte Chemie International Edition 49, 7632-7659 (2010).

5. Stewart, M. P. \& Buriak, J. M. Chemical and Biological Applications of Porous Silicon Technology. Advanced Materials 12, 859-869 (2000).

6. Lin, P. \& Yan, F. Organic Thin-Film Transistors for Chemical and Biological Sensing. Advanced Materials 24, 34-51 (2012).

7. Kauffman, D. R. \& Star, A. Carbon Nanotube Gas and Vapor Sensors, Angewandte Chemie International Edition 47, 6550-6570 (2008).

8. Das, A. et al. A nitrogen dioxide sensor based on an organic transistor constructed from amorphous semiconducting polymers. Advanced Materials 19, 4018-4023 (2007).

9. Kolmakov, A., Zhang, Y. X., Cheng, G. S. \& Moskovits, M. Detection of CO and $\mathrm{O}_{2}$ Using Tin Oxide Nanowire Sensors. Advanced Materials 15, 997-1000 (2003).

10. Barillaro, G., Lazzerini, G. M. \& Strambini, L. M. Modeling of porous silicon junction field effect transistor gas sensors: Insight into $\mathrm{NO}_{2}$ interaction. Applied Physics Letters 96, 162105-1/3 (2010).

11. Barillaro, G. \& Strambini, L. M. An integrated CMOS sensing chip for $\mathrm{NO}_{2}$ detection. Sensors and Actuators B 134, 585-590 (2008).

12. McAlpine, M. C., Ahmad, H., Wang, D. W. \& Heath, J. R. Highly ordered nanowire arrays on plastic substrates for ultrasensitive flexible chemical sensors. Nature Materials 6, 379-384 (2007). 
13. Engel, Y. et al. Supersensitive Detection of Explosives With Silicon Nanowire Arrays. Angewandte Chemie International Edition 49, 6830-6835 (2010).

14. Kong, J. et al. Nanotube Molecular Wires as Chemical Sensors. Science 287, 622-625 (2000).

15. Lu, G., Ocola, L. E. \& Chen, J. Room-Temperature Gas Sensing Based on Electron Transfer between Discrete Tin Oxide Nanocrystals and Multiwalled Carbon Nanotubes. Advanced Materials 21, 2487-2491 (2009).

16. Andringa, A.-M. et al. Gate-Bias Controlled Charge Trapping as a Mechanism for $\mathrm{NO}_{2}$ Detection with Field-Effect Transistors. Advanced Functional Materials 21, 100-107 (2011).

17. Zhang, D. H. et al. Detection of $\mathrm{NO}_{2}$ down to ppb levels using individual and multiple $\mathrm{In}_{2} \mathrm{O}_{3}$ nanowire devices, Nano Letters 4, 1919-1924 (2004).

18. Torsi, L. \& Dodabalapur, A. Organic Thin-Film Transistors as Plastic Analytical Sensors. Analitical Chemistry 77, 380A-387A (2005).

19. Hellmich, W., Müller, G., Braunmühl, Ch. Bosch-v., Doll, T. \& Eisele, I. Fieldeffect-induced gas sensitivity changes in metal oxides. Sensors and Actuators B Chemical 41, 132-139 (1997).

20. Fan, Z., Wang, D., Chang, P.-C., Tseng, W.-Y. \& Lu, J. G. ZnO Nanowire Field Effect Transistor and Oxygen Sensing Property. Applied Physics Letters 85, 5923-5925 (2004).

21. Zhang, Y., Kolmakov, A., Chretien, S. \& Metiu, H., Moskovits, M. Control of catalytic reactions at the surface of a metal oxide nanowire by manipulating electron density inside it. Nano Letters 4, 403-407 (2004).

22. Dattoli, E. N., Davydov, A. V. \& Benkstein, K. D. Tin oxide nanowire sensor with integrated temperature and gate control for multi-gas recognition. Nanoscale 4, 1760-1769 (2012).

23. Chen, G., Paronyan, T. M., Pigos, E. M. \& Harutyunyan, A. R. Enhanced gas sensing in pristine carbon nanotubes under continuous ultraviolet light illumination. Scientific Reports 2, 343 (2012).

24. Mubeen, S. \& Moskovits, M. Gate-Tunable Surface Processes on a SingleNanowire Field-Effect Transistor. Advanced Materials 23, 2306-2312 (2011).

25. Yaun, W., et al. Fault-tolerant dielectric elastomer actuators using single-walled carbon nanotube electrodes. Advanced Materials 20, 621-625 (2008).

26. Blaiszik, B. J., et al. Autonomic restoration of electrical conductivity. Advanced Materials 24, 398-401 (2012).

27. Tee, B. C.-K., Wang, C., Allen, R. \& Bao, Z. An electrically and mechanically selfhealing composite with pressure- and flexion-sensitive properties for electronic skin applications. Nature Nanotechnology 7, 825-832 (2012).
28. Tseng, T.-W., Huang, Y.-J. \& Li, J.-F. DABISR: A Defect-Aware Built-In SelfRepair Scheme for Single/Multi-Port RAMs in SoCs. IEEE Transactions on Computer-Aided Design of Integrated Circuits and Systems, 29, 1628-1639 (2010).

29. Mintarno, E., et al. Self-Tuning for Maximized Lifetime Energy-Efficiency in the Presence of Circuit Aging. IEEE Transactions On Computer-Aided Design Of Integrated Circuits And Systems 30, 760-773 (2011).

30. Sze, S. M. Physics of Semiconductor Devices, 2nd Edition, John Wiley and Sons (WIE), Wiley, November 18, 1981.

31. Barillaro, G., Lazzerini, G. M. \& Strambini, L. M. A porous silicon JFET gas sensor: Experimental and modeling. Proceedings of IEEE Sensors 4716485, 494 (2008).

32. Boarino, L. et al. Local environment of boron impurities in porous silicon and their interaction with $\mathrm{NO}_{2}$ molecules. Physical Review B 64, 205308-1/4 (2001).

\section{Ackwnoledgments}

Authors would like to thank staff at ST Microelectronics, Milan (Italy), for SEM images of PSJET.

\section{Author contributions}

G.B. conceived and designed the research. G.B. and G.M.L. worked out the theory. G.M.L. and L.M.S. performed the experiments. All authors analyzed the data and wrote the manuscript.

\section{Additional information}

Supplementary information accompanies this paper at http://www.nature.com/ scientificreports

Competing financial interests: The authors declare no competing financial interests. License: This work is licensed under a Creative Common Attribution-NonCommercial-ShareAlike 3.0 Unported License. To view a copy of this license, visit http://creativecommons.org/licenses/by-nc-sa/3.0/

How to cite this article: Lazzerini, G.M., Strambini, L.M. \& Barillaro, G. Addressing Reliability and Degradation of Chemitransistor Sensors by Electrical Tuning of the Sensitivity. Sci. Rep. 3, 1161; DOI:10.1038/srep01161 (2013). 